\title{
Effect of stage, comorbidities and treatment on survival among cancer patients with or without mental illness
}

Kristiina Manderbacka, Martti Arffman, Jaana Suvisaari, Aulikki Ahlgren-Rimpiläinen, Sonja Lumme, IImo Keskimäki and Eero Pukkala

\section{Background}

Earlier research suggests poorer outcome of cancer care among people with severe mental illness (SMI).

\section{Aims}

To assess the effect of stage at presentation, comorbidities and treatment on differences in survival among cancer patients with and without a history of SMI in Finland.

\section{Method}

The total population with a first cancer diagnosis in 19902013 was drawn from the Finnish Cancer Registry. Hospital admissions because of SMI and deaths were obtained from administrative registers. We calculated Kaplan-Meier estimates and Cox regression models to examine survival differences.

\section{Results}

We found excess mortality in people with a history of psychotic and substance use disorders. Cancer stage and comorbidity did not explain mortality differences. Controlling for cancer treatment decreased the differences. The mortality gap between patients with psychosis and cancer patients without SMI increased over time.

\section{Conclusions}

Integrated medical and psychiatric care is needed to improve outcomes of cancer care among patients with SMI.

\section{Declaration of interest}

None.

\section{Copyright and usage}

(c) The Royal College of Psychiatrists 2017.
Several recent studies suggest that the incidence of cancer in people with severe mental illness (SMI) may not be elevated, ${ }^{1-3}$ and study results differ concerning cancer stage at presentation as some studies have found only few differences in stage at presentation ${ }^{4}$ and some report presentation at a later stage among people with SMI. ${ }^{2}$ Patients with a psychiatric condition have further been reported to have a reduced likelihood of surgery, and to have received less radiotherapy and fewer chemotherapy sessions. ${ }^{2}$ Additionally, most groups of people with SMI have been reported to have worse cancer survival not explained by stage at presentation. ${ }^{1-5}$ Similar results have been reported concerning specific cancers, including breast cancer, ${ }^{6,7}$ colorectal cancer, ${ }^{6}$ oral cancer, ${ }^{8}$ pancreatic adenocarcinoma ${ }^{9}$ and prostate cancer. ${ }^{10}$ However, earlier studies are mainly based on regional samples and few of them examine both all cancers and history of psychosis, substance use disorders and mood disorders. The aim of the current study was to assess whether cancer stage at presentation, comorbidities or treatment received had an effect on differences in survival of people with cancer with and without history of mental illness taking into account gender, age and year of cancer diagnosis in Finland in 1990-2013. We hypothesised that (a) patients with SMI have higher case fatality from cancer than patients without SMI, (b) this is particularly true for patients with psychotic and substance use disorders but less pronounced in mood disorders, and (c) this is caused by both presentation at a more advanced stage and less intensive treatment.

\section{Method}

\section{Population with a first cancer diagnosis in 1990-2013}

The population with their first cancer diagnosis in 1990-2013 was obtained from the National Cancer Registry including all cancer patients in Finland. The data were examined back until 1969 to ensure that no earlier cancer diagnoses could be found. We focused on the four most prevalent cancers, namely cancer of the lung (C34), breast (C50) colon and rectum (C18, C19.8, C20-C21) and prostate (C61). Additionally, cancers were classified with the top ten cancers separately for men and women and other cancers (the rest of the C-diagnoses) were included as one group. Information concerning hospital admissions because of severe mental illness (SMI) before cancer diagnosis was individually linked to the study population from the Hospital Discharge Register for the years 1969-2013. We defined three main categories of mental disorders, namely substance use disorder (ICD-10 code F10-19 and corresponding ICD-9 and ICD-8 codes), ${ }^{11-13}$ psychosis (F20, F22-29) and mood disorders (F30-33, F38-39) as the main diagnosis for hospital admission 1 year before the cancer diagnosis or earlier. If an individual had been admitted to hospital more than once and given a diagnosis belonging to more than one SMI category, we hierarchically allocated the individual to the psychosis subpopulation if diagnosed with psychosis at least once. Patients with substance use related and mood disorder related diagnoses were allocated to the substance use group. These individuals remained in the SMI population since their first admission to hospital as a result of the long natural course of these mental disorders. The data linkages were performed by the competent authorities and the study group received anonymised data.

\section{Psychiatric and oncological services in Finland}

The Finnish healthcare system is mainly public and financed by taxes collected by the state and the municipalities, including all psychiatric hospital services. ${ }^{14}$ A de-institutionalisation of psychiatric services has occurred in Finland since the beginning of 1980 s, with a substantial reduction in psychiatric hospital beds and an increase in out-patient services. Municipalities are responsible for organising out-patient services, which are provided by public, private and third-sector actors. ${ }^{15}$ Services for substance 
use disorders are provided within both social welfare and healthcare and by municipal, private and third-sector actors. ${ }^{16}$ Although the diagnostic process in cancers usually begins in primary healthcare, and first diagnostic tests are run in primary care, a preliminary diagnosis of cancer leads to referral to specialist care. ${ }^{17}$ Oncological services in Finland are mainly provided in specialist care organised in 20 hospital districts supplying specialist services for the residents. The hospital districts are managed and funded by the municipalities. The number of private clinics in oncological care is very small, for example in our data $0.5 \%$ of patients with cancer had been treated in private clinics.

\section{Covariates in the models}

Comorbidity was defined by admission to hospital for particular diseases (congestive heart failure, peripheral vascular disease, cerebrovascular disease, dementia, chronic pulmonary disease, rheumatic disease, peptic ulcer disease, liver disease, diabetes with or without chronic complications, hemiplegia or paraplegia, and renal disease) in the Hospital Discharge Register for 2 years preceding cancer incidence using a modified Charlson's comorbidity index. ${ }^{18}$ Cancer stage at presentation was categorised as (a) localised (51\% of individuals, overall), (b) metastasised (regional or distant, 33\%), and (c) unknown (16\%). Cancer treatment was measured by six variables: (a) surgical therapy, (b) radiotherapy (both of which were further categorised as curative or palliative), (c) chemotherapy, (d) hormonal therapy, (e) other, and (f) treatment unknown. Age at presentation was categorised in 5-year age groups and year of cancer diagnosis as a continuous variable. Information concerning mortality was obtained from the Finnish Causes of Death statistics. We examined both cancer-specific and all-cause mortality. Ethical approval for the study was received from the Research Ethics Committee of the National Institute for Health and Welfare.

\section{Statistical methods}

We calculated proportions of SMI among incident cancer patients. We then calculated Kaplan-Meier estimates to examine survival differences between patient groups among men and women. Cox regression models were used to study survival differences in four steps to study the impact of covariates on patient group differences and survival in the whole study period. In the first step, we estimated hazard ratios for patient groups adjusting for age, year of incidence from 1990 and cancer type. In the following steps, we further adjusted in the second step for stage at presentation, in the third for cancer treatment, and in the fourth for Charlson's comorbidity index. Generalised $R^{2}$ was used as a measure of how well each set of covariates predicts survival. ${ }^{19}$ We conducted further Cox regression analysis to examine if patient group differences remained the same during the study period by adding the interaction term for incidence year and patient group and extracted average annual change estimates for each of the patient groups. In order to estimate hazard ratios for equivalent potential follow-up in different time points, we adjusted Cox regression models with 5-year follow-up in three cancer incidence periods (1990-1994, 1997-2001 and 20042008). SAS version 9.3 (SAS Institute, Cary, NC) was used in statistical analyses of the study.

\section{Results}

There were altogether 628435 cases of first cancers in 1990-2013 in Finland. The number of women was larger in the cancer population than that of men, as $53 \%$ of our cancer population was female. Among men, the most common SMI was substance use disorder, whereas among women mood disorders and psychoses were more common (Table 1). Patients with a history of substance use disorder were younger than other cancer patients, and together with patients with a history of psychosis more often had a metastasised cancer at presentation among both men and women. Comorbidities were more common among men, but there were few differences between patient groups in Charlson's comorbidity indices.

Among men the top-10 cancers were cancer of the prostate (ICD-10 code C61), skin (C44), lung (C34), colon and rectum $(\mathrm{C} 18, \mathrm{C} 20)$, cancers of the haematopoietic and reticuloendothelial system (C42), bladder (C67), stomach (C16), pancreas (C25), kidney (C64) and brain (C71). Among women, the top-10 cancers were cancer of the breast (C50), skin (C44), colon and rectum (C18, C20), cervix uteri (C53), corpus uteri (C54), ovaries (C56), lung (C34), cancers of the haematopoietic and reticuloendothelial system (C42), pancreas (C25) and stomach (C16).

The Kaplan-Meier curves in Fig. 1 indicate that cancer-specific mortality was higher among men with a history of psychosis and substance use disorder, whereas there were only small differences between those with a mood disorder history and those without a history of SMI. Among women a stepwise gradient was found from those without SMI through mood disorders and substance use disorders to those with psychosis. Additionally, among both men and women with psychosis and substance use disorders excess mortality was found already, during the first years after cancer diagnosis.

Table 2 presents the risk of cancer-specific mortality among the four patient groups and the mortality risk when taking into

Table 1 Basic background characteristics of the 1990-2013 population with a first cancer in Finland by gender and history of severe mental illness (SMI)

\begin{tabular}{|c|c|c|c|c|c|c|c|c|}
\hline & \multicolumn{4}{|c|}{ Men } & \multicolumn{4}{|c|}{ Women } \\
\hline & $\begin{array}{c}\text { Substance } \\
\text { misuse }\end{array}$ & Psychosis & $\begin{array}{c}\text { Mood } \\
\text { disorder }\end{array}$ & No SMI & $\begin{array}{l}\text { Substance } \\
\text { misuse }\end{array}$ & Psychosis & $\begin{array}{c}\text { Mood } \\
\text { disorder }\end{array}$ & No SMI \\
\hline$n$ & 13165 & 5325 & 4367 & 273599 & 3058 & 7988 & 7793 & 313140 \\
\hline$\%$ & 4.4 & 1.8 & 1.5 & 92.3 & 0.9 & 2.4 & 2.4 & 94.3 \\
\hline Age, mean & 64.8 & 64.4 & 67.3 & 67.6 & 60.0 & 65.8 & 67.6 & 64.7 \\
\hline Breast cancer, \% & - & - & - & - & 25 & 29 & 24 & 25 \\
\hline Prostate cancer, $\%$ & 21 & 16 & 25 & 26 & - & - & - & - \\
\hline Colorectal cancer, \% & 7 & 7 & 7 & 8 & 5 & 6 & 6 & 7 \\
\hline Lung cancer, \% & 21 & 23 & 13 & 11 & 11 & 6 & 5 & 3 \\
\hline Other, \% & 51 & 54 & 55 & 56 & 59 & 58 & 64 & 65 \\
\hline Metastasised at presentation, $\%$ & 42 & 42 & 34 & 33 & 39 & 39 & 33 & 32 \\
\hline Charlson's index, mean & 0.19 & 0.19 & 0.21 & 0.21 & 0.13 & 0.15 & 0.16 & 0.15 \\
\hline
\end{tabular}


(a)

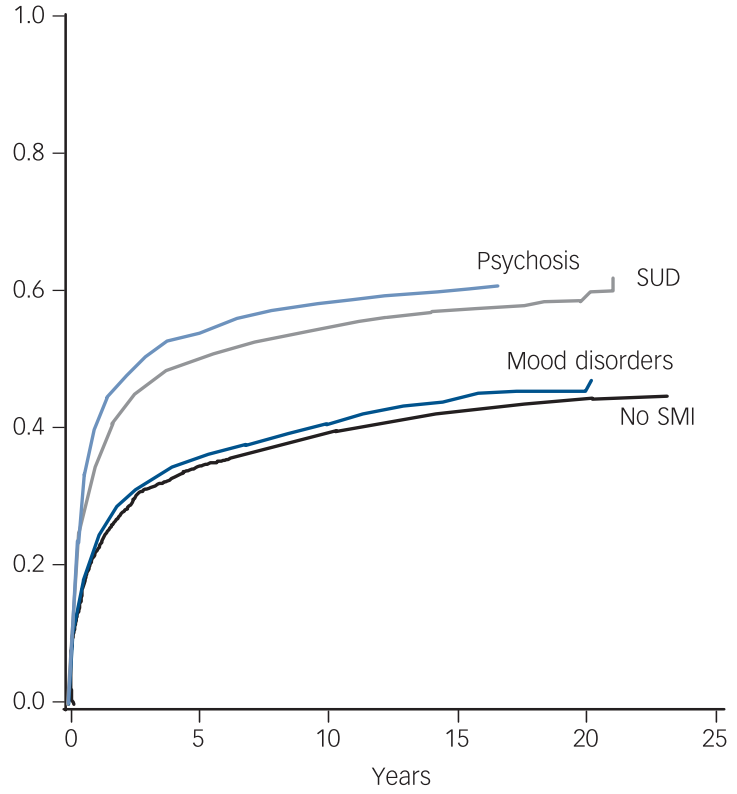

(b)

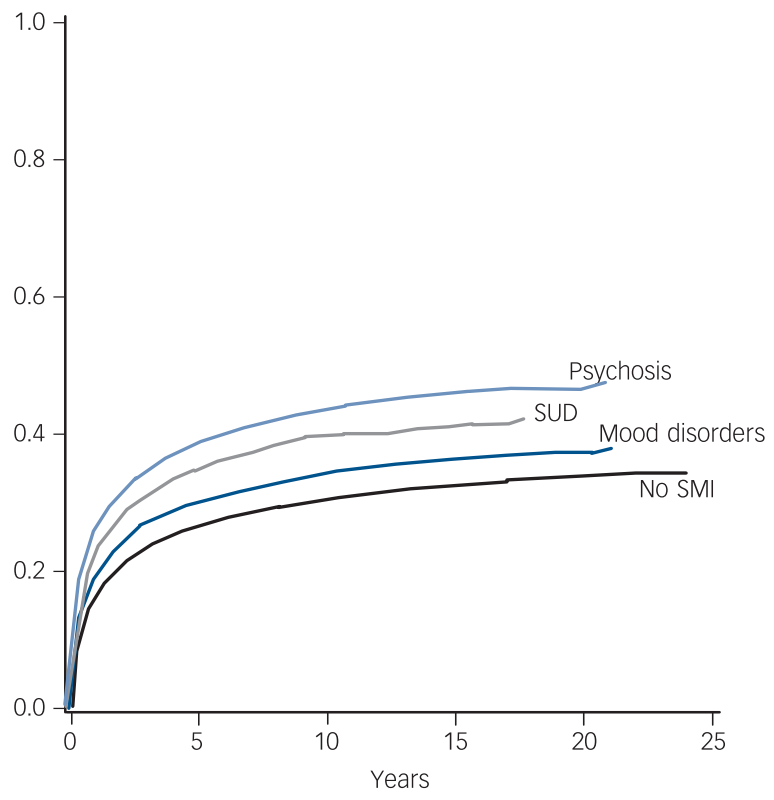

Fig. 1 Kaplan-Meier curves for the distribution of cancer-specific mortality (cumulative distribution function) among Finnish (a) men and (b) women diagnosed with their first cancer in 1990-2013.

SMI, severe mental illness; SUD, substance use disorder.

account stepwise age, cancer type and year of cancer diagnosis, cancer stage at presentation, cancer treatment and comorbidities. When taking into account only age, year of cancer diagnosis and cancer type, all groups with a history of SMI had higher mortality risk compared with those without a history of SMI. The excess mortality risk was especially high among those with history of psychosis both among men and among women. Although all the examined factors were important predictors of mortality overall, the only factor that had an effect on differences between patient groups was cancer treatment. Taking into account cancer treatment (model III) decreased the mortality risk among those with a history of psychosis and substance use problems. Findings regarding all-cause mortality were similar (online Table DS1).

When examining the average annual decrease in cancerspecific mortality, we found that the decrease was $3.1 \%$ (95\% CI 3.0-3.2) among men and 3.2\% (3.1-3.3) among women without a history of severe mental disorders. Among men with a history of psychosis or substance use disorder the decrease was significantly slower; $1.7 \% \quad(1.1-2.3)$ and $2.5 \% \quad(2.1-2.8)$, respectively. Among women a similar difference was found only among those with history of psychosis; among them the average annual decrease was $2.2 \%(1.6-2.7)$.
Table 3 presents the 5 -year cancer-specific mortality risk of incident cancer patients in three time periods. The interaction between period and SMI type was statistically significant among women $(P=0.0091)$; among patients with a history of psychosis the relative excess mortality risk (compared with patients with no history of SMI) increased more than among other SMI groups. Among men, the pattern was similar, but the interaction was not statistically significant $(P=0.0649)$. Cancer-specific mortality risk was higher among patients with a history of psychosis or substance use disorders compared with those without a history of mental disorders in each three periods among men and women. The risk was not consistent among patients with a history of mood disorder, but the risk was significantly higher in 2004-2008 compared with patients without a history of mental disorders.

In the group having their first cancer episode in 1997-2001, the mortality risk increased among those with a history of psychosis, both among men $(P=0.0234)$ and among women $(P=0.0411)$, compared with the $1990-1994$ period. The mortality risk further increased in this patient group among those with their first cancer in 2004-2008 compared with the 1990-1994 period (among men $P=0.0012$; among women $P=0.0022$ ). Whereas

\begin{tabular}{|c|c|c|c|c|}
\hline & Model I & Model II & Model III & Model IV \\
\hline \multicolumn{5}{|l|}{ Men } \\
\hline Substance misuse, HR $(95 \% \mathrm{Cl})$ & $1.38(1.34-1.41)$ & $1.38(1.35-1.42)$ & $1.31(1.28-1.35)$ & $1.31(1.28-1.35)$ \\
\hline Psychosis, HR (95\% Cl) & $1.59(1.53-1.65)$ & $1.59(1.53-1.65)$ & $1.41(1.36-1.47)$ & $1.42(1.36-1.47)$ \\
\hline Mood disorder, HR (95\% Cl) & $1.09(1.04-1.15)$ & $1.11(1.06-1.17)$ & $1.08(1.03-1.14)$ & $1.08(1.03-1.14)$ \\
\hline Generalised $R^{2}$ & 0.3927 & 0.4662 & 0.5130 & 0.5131 \\
\hline \multicolumn{5}{|l|}{ Women } \\
\hline Substance misuse, HR $(95 \% \mathrm{Cl})$ & $1.44(1.36-1.53)$ & $1.43(1.34-1.51)$ & $1.37(1.28-1.45)$ & $1.36(1.28-1.45)$ \\
\hline Psychosis, HR (95\% Cl) & $1.64(1.58-1.70)$ & $1.61(1.55-1.66)$ & $1.47(1.42-1.53)$ & $1.47(1.42-1.53)$ \\
\hline Mood disorder, HR (95\% Cl) & $1.11(1.07-1.16)$ & $1.12(1.08-1.17)$ & $1.10(1.05-1.14)$ & $1.10(1.05-1.14)$ \\
\hline Generalised $R^{2}$ & 0.3720 & 0.4452 & 0.4906 & 0.4906 \\
\hline
\end{tabular}




\begin{tabular}{|c|c|c|c|}
\hline \multirow[b]{2}{*}{ Year of cancer incidence } & \multicolumn{3}{|c|}{$\mathrm{HR}(95 \% \mathrm{Cl})$} \\
\hline & 1990-1994 & 1997-2001 & 2004-2008 \\
\hline \multicolumn{4}{|l|}{ Men } \\
\hline Substance misuse & $1.29(1.21-1.39)$ & $1.30(1.24-1.39)$ & $1.33(1.26-1.41)$ \\
\hline Psychosis & $1.22(1.11-1.34)$ & $1.41(1.30-1.53)$ & $1.50(1.38-1.63)$ \\
\hline Mood disorder & $1.10(0.95-1.26)$ & $1.06(0.94-1.18)$ & $1.13(1.02-1.26)$ \\
\hline \multicolumn{4}{|l|}{ Women } \\
\hline Substance misuse & $1.35(1.09-1.66)$ & $1.29(1.13-1.48)$ & $1.62(1.43-1.82)$ \\
\hline Psychosis & $1.34(1.22-1.46)$ & $1.51(1.40-1.64)$ & $1.61(1.49-1.73)$ \\
\hline Mood disorder & $1.17(1.04-1.31)$ & $1.08(0.99-1.18)$ & $1.10(1.01-1.20)$ \\
\hline
\end{tabular}

the hazard ratio also increased among women with a history of substance use problems, the change was not statistically significant.

\section{Discussion}

\section{Main findings and comparison with findings from other studies}

In this study we examined the effect of a history of SMI on mortality among a total population of people with their first cancer episode in 1990-2013. Our study adds to the literature by examining all cancers and history of psychosis, substance use disorders and mood disorders simultaneously using nationwide register data. In line with earlier research, we found excess mortality especially among individuals with a history of psychotic and substance use disorders. ${ }^{1-5}$ Cancer was more often detected at a metastasised stage in those with a history of psychotic and substance use disorders than in individuals with a mood disorder or no SMI history. However, although stage at presentation was an important predictor of mortality, it had little effect on differences in mortality. In general, earlier studies have reported that patients with mental disorders present at a later stage, receive less treatment in terms of surgery and chemotherapy, and, taking into account stage at presentation, have poorer survival. ${ }^{1-5,8,9}$ In our study, taking into account differences in treatment did decrease the differences but did not abolish them, and taking into account comorbidity did not have an effect on the differences.

Consistent with many previous studies, psychotic and substance use disorders were associated with larger excess mortality than mood disorders, ${ }^{2,4}$ although in some studies mortality risk has been similar in schizophrenia and depressive disorders $^{5}$ or even higher in mood disorders. ${ }^{1,20}$ However, it should be pointed out that because of the hierarchical diagnostic classification in our study the mood disorder group did not include patients with comorbid substance use disorders. In previous studies, comorbid substance use disorders have contributed to excess mortality from cancer in patients with mood disorders. ${ }^{21}$ Lung cancer was more common in men with a history of psychotic and substance use disorders and in women with substance use disorder compared with the other groups, which is consistent with the high rates of smoking in these patient groups. ${ }^{22,23}$ However, cancer type was adjusted for in our analyses. Although comorbidity with various chronic conditions is more common in people with SMI than in the general population ${ }^{24,25}$ our results showed no difference in Charlson's comorbidity index between cancer patients with or without SMI, and comorbidity did not explain excess cancer mortality in the SMI groups.

An alarming finding was that the cancer-specific mortality gap between patients with a history of psychosis who had cancer and other cancer patients increased over time. This is consistent with findings on a worsening mortality gap in people with schizophrenia in general. ${ }^{26}$ The increasing mortality gap among people with the most severe forms of mental illness and cancer compared with people without a history of mental illness is likely to partly reflect differences in quality of cancer treatment.

Poorer quality of cancer treatment in patients with schizophrenia is a consistent finding in previous literature. ${ }^{2}$ Barriers in cancer care among people with SMI have not been studied in Finland but a recent international review identified patient-level, provider-level and systems-level factors contributing to this disparity in cancer treatment in patients with schizophrenia. ${ }^{27}$ Stigma toward patients with schizophrenia may affect treatment decisions and create barriers to optimal care. ${ }^{27}$ There is also evidence that patients with schizophrenia experience barriers to clinical trials including challenges with trust and high medical comorbidity. They may also have more difficulties in navigating the healthcare system. Social factors including social isolation and lack of social support are also important factors likely to contribute to diagnostic delay and treatment differences. ${ }^{27}$ Patients with schizophrenia may also have cognitive deficits and problems in communication, have difficulties agreeing with more intensive treatment regimens and may not be able to complete these treatments. It is therefore important to allow more time for their appointments, to engage family members or caregivers in the treatment and to arrange home nursing services or other assistance during cancer treatment. Collaboration between mental healthcare and oncological care is crucial for the optimal cancer care for these patients, ${ }^{27}$ but in practice it is often limited or lacking.

The quality of cancer care in patients with substance use disorders has received little attention. Substance use disorders are associated with comorbidities that affect cancer treatment decisions and options ${ }^{28}$ but the possible role of stigma has received little attention. The possible role of treatment adherence in the poorer prognosis of cancer in patients with substance use and mood disorders should be studied further. As in schizophrenia, collaboration between mental health or substance use services and oncological care is needed to ensure optimal cancer care for these patients.

Administrative databases contain only limited information on the diagnosis and treatment process. Previous studies point to multiple problems: less frequent participation in cancer screening, delays in the diagnosis of cancer, difficulties in access to different treatments but also increased risk of side-effects because of interactions with psychotropic medications, to name a few. ${ }^{27,29}$ Further studies including detailed clinical information are needed to address these issues comprehensively.

\section{Methodological considerations}

The strength of our study was the use of nationwide, unselective representative data concerning the total population of Finnish 
residents with their first cancer diagnosis in 1990-2013 collected from the Finnish Cancer Registry and Hospital Discharge Register covering the total population and based on clinical diagnoses. Notification of cancer to the Cancer Registry is mandatory in Finland and a good comparability and validity has been documented in the Cancer Registries of all the Nordic countries in a survey comparing the practices of the registries. ${ }^{30}$ Close to $100 \%$ coverage of incident cases has been reported in the Finnish Cancer Registry and the accuracy of the records has been found to be high. ${ }^{31}$ We were also able to include patients with a history of substance use disorders from the Hospital Discharge Register. The accuracy and coverage of the register has, in general, been reported to be good. ${ }^{32}$ The Finnish Causes of Death statistics are considered valid and reliable by international standard, thus enabling us to study cancer-specific mortality. ${ }^{33}$ Furthermore, the rate of confirmation of the diagnosis by autopsy in Finland is high compared with many other countries (in 2014, 23\% for all deaths and 53\% of those under 65 years of age). ${ }^{34}$

The benefit of using a register-based total population of incident cancer patients leads to having no missing data or selection bias in the analysis as would be the case in, for example, hospital populations. In our study people with less severe mental disorders who have not been admitted to hospital are excluded from the SMI population. Although most patients with psychotic disorder are admitted to hospital at some point in their illness, ${ }^{35}$ hospital-treated substance use disorder and mood disorders capture only the most severe forms of these disorders. In our study, we were able to define a group of individuals with SMI who had a definite history of contact with healthcare and a diagnosis confirmed by a psychiatrist. A weakness of administrative registers is that they do not contain information about health behaviours, disease severity or data on the treatment process. Another weakness in our study is that we could not analyse bipolar disorder and depressive disorders separately because of the small numbers of patients with bipolar disorder. We could, however, examine the Kaplan-Meier survival curves of these patient groups and found no differences in them.

\section{Policy implications}

People with SMI have increased mortality from cancer compared with other cancer patients, and this seems mainly to be because of the poorer quality of cancer treatment. Different models of integrated medical and psychiatric care and involving professionals responsible for the treatment of SMI in the cancer treatment plan at the time of diagnosis helps to improve this situation. However, further studies should collect more in-depth information on the whole cancer treatment process in order to identify the best opportunities to improve cancer treatment in people with SMI.

\section{Funding}

This study was partly funded by the Cancer Society in Finland, but the Society had no involvement in its design, data collection, findings or decision to publish.

Kristiina Manderbacka, PhD, Martti Arffman, MSc, National Institute for Health and Welfare, Health and Social Systems Research, Helsinki, Finland; Jaana Suvisaari, PhD, Aulikki Ahlgren-Rimpiläinen, PhD, National Institute for Health and Welfare, Mental Health, Helsinki, Finland; Sonja Lumme MSc, National Institute for Health and Welfare, Health and Social Systems Research, Helsinki, Finland; Imo Keskimäki, PhD, National institute for Heath and Wefar PhD, National instite for Health and Wearch, Hel Healh, Find Helsinki, Finland and Faculty of Social Sciences, University of Tampere, Finland; Eero Pukkala, PhD, Finnish Cancer Registry, Institute for Statistical and Epidemiologica Cancer Research, Helsinki, and Faculty of Social Sciences, University of Tampere, Finland

Correspondence: Kristiina Manderbacka, Health and Social Systems Research National Institute for Health and Welfare, PO Box 30, 00271 Helsinki, Finland. Email: kristiina.manderbacka@thl.fi

First received 6 Feb 2017, final revision 27 Jun 2017, accepted 3 Jul 2017

\section{References}

1 Lawrence D, Holman CD, Jablensky AV, Threlfall TJ, Fuller SA. Excess cancer mortality in Western Australian psychiatric patients due to higher case fatality rates. Acta Psychiatr Scand 2000; 101: 382-8.

2 Kisely S, Crowe E, Lawrence D. Cancer-related mortality in people with mental illness. JAMA Psychiatry 2013; 70: 209-17.

3 Kisely S, Sadek J, MacKenzie A, Lawrence D, Campbell LA. Excess cancer mortality in psychiatric patients. Can J Psychiatry 2008; 53: 753-61.

4 Chang C-K, Hayes RD, Broadbent MTM, Hotopf M, Davies E, Møller $\mathrm{H}$, et al. A cohort study on mental disorders, stage of cancer at diagnosis and subsequent survival. BMJ Open 2014; 4: e004295.

5 Batty GD, Whitley E, Gale CR, Osborn D, Tynelius P, Rasmussen F. Impact of mental health problems on case fatality in male cancer patients. $\mathrm{Br} J$ Cancer 2012; 106: 1842-5.

6 Cunningham R, Sarfati D, Stanley J, Peterson D, Collings S. Cancer survival in the context of mental illness: a national cohort study. Gen Hosp Psychiatry 2015; 37: 501-6.

7 Goodwin JS, Zhang DD, Ostir GV. Effect of depression on diagnosis, treatment, and survival of older women with breast cancer. J Am Geriatr SoC 2004; 52: 106-11.

8 Chang TS, Hou SJ, Su YC, Chen LF, Ho MC, Lee MS, et al. Disparities in oral cancer survival among mentally ill patients. PLOS One 2013; 8: e70883.

9 Boyd CA, Benarroch-Gampel J, Sheffield KM, Han Y, Kuo YF, Riall TS The effect of depression on stage at diagnosis, treatment, and survival in pancreatic adenocarcinoma. Surgery 2012; 152: 403-13.

10 Safdieh JJ, Schwartz D, Rineer J, Weiner JP, Wong A, Schreiber D. Does the presence of major psychiatric disorder affect tolerance and outcomes in men with prostate cancer receiving radiation therapy? Am J Mens Health 2017; 11: $5-12$.

11 World Health Organization. The ICD-10 Classification of Mental and Behavioural Disorders: Clinical Descriptions and Diagnostic Guidelines. WHO, 1992.

12 World Health Organization. International Statistical Classification of Diseases and Related Health Problems (ICD-9). WHO, 1978.

13 World Health Organization. International Statistical Classification of Diseases and Related Health Problems (ICD-8). WHO, 1967.

14 Lehtinen V, Taipale V. Integrating mental health services: the Finnish experience. Int J Integr Care 2001; 1: e26.

15 Ala-Nikkola T, Sadeniemi M, Kaila M, Saarni S, Kontio R, Pirkola S, et al. How size matters: exploring the association between quality of mental health services and catchment area size. BMC Psychiatry 2016; 16: 289.

16 Varjonen V. Finland - Drug Situation 2014. National Institute for Health and Welfare, 2015 (http://urn.fi/URN:ISBN:978-952-302-427-4).

17 Hermanson $\mathrm{T}$, vertio $\mathrm{H}$, Mattson J. Development of Cancer Treatment in 2010-2020. Working Group Report. Ministry of Social Affairs and Health, 2010 (http://urn.fi/URN:ISBN:978-952-00-2971-5).

18 Quan $H$, Sundararajan V, Halfon $\mathrm{P}$, Fong A, Burnand B, Luthi JC, et al. Coding algorithms for defining comorbidities in ICD-9-CM and ICD-10 administrative data. Med Care 2005; 43: 1130-9.

19 Allison PD. Survival Analysis Using the SAS System: A Practical Guide. SAS Institute, 1995.

20 Pinquart M, Duberstein PR. Depression and cancer mortality: a metaanalysis. Psychol Med 2010; 40: 1797-810.

21 Crump C, Sundquist K, Winkleby MA, Sundquist J. Comorbidities and mortality in bipolar disorder: a Swedish national cohort study. JAMA Psychiatry 2013; 70: 931-9.

22 Guydish J, Passalacqua E, Pagano A, Martinez C, Le T, Chun J, et al. An international systematic review of smoking prevalence in addiction treatment. Addiction 2016; 111: 220-30

23 Partti K, Vasankari T, Kanervisto M, Perälä J, Saarni SI, Jousilahti P, et al. Lung function and respiratory diseases in people with psychosis: population-based study. Br J Psychiatry 2015; 207: 37-45.

24 Crump C, Winkleby MA, Sundquist $K$, Sundquist J. Comorbidities and mortality in persons with schizophrenia: a Swedish national cohort study. Am J Psychiatry 2013; 170: 324-33.

25 Crump C, Sundquist $K$, Winkleby MA, Sundquist J. Comorbidities and mortality in bipolar disorder: a Swedish national cohort study. JAMA Psychiatry 2013; 70: 931-9.

26 Saha S, Chant D, McGrath J. A systematic review of mortality in schizophrenia: is the differential mortality gap worsening over time? Arch Gen Psychiatry 2007; 64: 1123-31. 
27 Irwin KE, Henderson DC, Knight HP, Pirl WF. Cancer care for individuals with schizophrenia. Cancer 2014; 120: 323-34.

28 Pinter M, Trauner M, Peck-Radosavljevic M, Sieghart W. Cancer and liver cirrhosis: implications on prognosis and management. ESMO Open 2016; 1 e000042.

29 Howard LM, Barley EA, Davies E, Rigg A, Lempp H, Rose D, et al. Cancer diagnosis in people with severe mental illness: practical and ethical issues. Lancet Oncol 2010; 8: 797-804.

30 Association of the Nordic Cancer Registeries. Survey of Nordic Cancer Registries. Danish Cancer Society, 2000

31 Korhonen P, Malila N, Pukkala E, Teppo L, Albanes D, Virtamo J. The Finnish Cancer Registry as follow-up source of a large trial cohort. Acta Oncologica 2002; 41: 381-8.
32 Sund R. Quality of the Finnish Hospital Discharge Register: a systematic review. Scan J Public Health 2012; 40: 505-15.

33 Lahti R. From Findings to Statistics: An Assessment of Finnish Medical Cause-of-Death Information in Relation to Underlying-Cause Coding. Department of Forensic Medicine, University of Helsinki, 2005.

34 StatFin database. Autopsies by Age in 1975-2014. Statistics Finland, 2016 (http://pxnet2.stat.fi/PXWeb/pxweb/fi/StatFin/StatFin_ter_ksyyt/?table list $=$ true \& $r x i d=$ b94b307b-a1ad-4fed-9ffo-29b3bddd02e4).

35 Suvisaari J, Perälä J, Saarni SI, Juvonen $\mathrm{H}$, Tuulio-Henriksson A, Lönnqvist J. The epidemiology and descriptive and predictive validity of DSM-IV delusional disorder and subtypes of schizophrenia. Clin Schizophr Relat Psychoses 2009; 2: 289-97.

\title{
A Monster Calls - dissociation in children's literature
}

\author{
Nishan Ghoshal, Paul O. Wilkinson
}

A Monster Calls is a children's book written by Patrick Ness and published in 2011. It has received numerous awards, and a recent film adaptation has also garnered critical acclaim.

The book tells the story of Conor O'Malley, a 13-year-old boy whose mother is terminally ill. Over the course of the book, Conor is repeatedly visited by a monster who tells him different stories. These stories are reflections of fears and worries that Conor is experiencing in his everyday life. After he has 'come around' from these 'visits', conor sees that somebody has engaged in destructive and violent behaviour, such as trashing his grandmother's lounge or assaulting a bully. Conor's memories are that he has seen the monster carry out these acts, even though the witnesses state it was Conor.

The story ends with Conor telling a story of his own, a recurrent nightmare in which his mother is dangling off a cliff edge and Conor is grabbing hold of her by her arm. Eventually, his mother's weight becomes too much to bear and he lets go, letting her fall. The monster helps him understand that it is all right for him to feel overwhelmed and worn-out by his mother's illness, and the fact that he has let go does not make him a bad son.

Assuming the monster is not real, Conor's actions throughout the book serve as a good example of dissociation. Conor displays this at numerous points by believing that it is not him acting, but the monster (external and independent from his self). During these events, Conor experiences feelings of dissociation and a sense of disconnect from himself; he describes the events as if he is watching from an outsider's perspective, seeing the 'monster' act in his stead.

The aetiology behind Conor's dissociation lies in the emotional trauma he deals with. As he finds it hard to acknowledge and express his feelings about his mother's terminal illness, they manifest as dissociative symptoms. Another precipitating factor is that Conor faces recurrent bullying at school and has few friends - indeed, one of the stories the monster tells reflects on the fact that conor feels invisible in his school. This feeling of invisibility may serve as another factor that drives his sense of dissociation. Furthermore, he has little contact with his father (who lives in another country) and is often taken care of by his grandmother, whom he does not like.

A Monster Calls is an unusual book in the genre of children's fiction in that it deals with distressing topics such as terminal illness and bereavement. By portraying the subject in a sensitive and suitable manner, however, it provides an appealing experience to the younger reader who may be dealing with similar issues. The dissociation that Conor experiences is an engaging example of how feelings of disconnect can act as a coping mechanism during times of emotional trauma. 